\title{
The disconnection technique with the use of a bronchial blocker for improving nonventilated lung collapse in video-assisted thoracoscopic surgery
}

\author{
Qian Cheng ${ }^{1,2 \#}$, Zhiyong He ${ }^{1,2 \#}$, Ping Xue ${ }^{1,2}$, Qianyun Xu ${ }^{1,2}$, Minmin Zhu ${ }^{1,2,3}$, Wankun Chen ${ }^{1,2,3}$, \\ Changhong Miao ${ }^{1,2,3}$
}

${ }^{1}$ Department of Anesthesiology, Fudan University Shanghai Cancer Center, Shanghai 200032, China; ${ }^{2}$ Department of Oncology, Shanghai Medical College, Fudan University, Shanghai 200032, China; ${ }^{3}$ Department of Anesthesiology, Zhongshan Hospital, Fudan University, Shanghai 200032, China

Contributions: (I) Conception and design: C Miao, W Chen; (II) Administrative support: C Miao; (III) Provision of study materials or patients: Q Cheng, Z He; (IV) Collection and assembly of data: P Xue, Q Xu, Z He; (V) Data analysis and interpretation: W Chen, Q Cheng, M Zhu; (VI) Manuscript writing: All authors; (VII) Final approval of manuscript: All authors.

\#These authors contributed equally to this work.

Correspondence to: Changhong Miao, MD, PhD; Wankun Chen, MD, PhD; Minmin Zhu, MD, PhD. Department of Anesthesiology, Fudan University Shanghai Cancer Center and Department of Oncology, Shanghai Medical College, Fudan University, 270\# Dong-An Road, Shanghai 200032, China; Department of Anesthesiology, Zhongshan Hospital, Fudan University, 180\# Feng-Lin Road, Shanghai 200032, China. Email: miaochangh@163.com; chenwank@163.com; zhu_mm@126.com.

Background: One-lung ventilation (OLV) is becoming an essential component of thoracic anesthesia. The two principal devices used for OLV are a double-lumen tube (DLT) and a bronchial blocker (BB). We hypothesized that the use of a BB with the disconnection technique would improve the quality of lung collapse in video-assisted thoracoscopic surgery (VATS).

Methods: Seventy-five patients undergoing scheduled VATS were enrolled in this study and were randomly divided into two groups: a left-sided DLT group (Group D) and a BB with the disconnection technique group (Group B). OLV was initiated when the surgeon performed the skin incision. In Group D, the left channel of the DLT was opened to the air. In Group B, the lung was deflated via the disconnection technique, thus opening the breathing circuit to the air fifteen seconds after opening the pleura. The mean arterial pressure (MAP) and heart rate (HR) during induction; the quality of lung collapse 1 and 10 minutes after pleural opening; the time required for complete lung collapse; the correct placement of the device; and the number of patients suffering from a sore throat after surgery were recorded.

Results: Compared with the use of the DLT, the use of the BB with the disconnection technique was associated with a similar quality of lung collapse, a comparable required time for total lung collapse $(\mathrm{P}>0.05$, respectively), a lower incidence of sore throat both when leaving the PACU and 24 hours after surgery $(34.2 \%$ vs. $13.5 \%, 15.8 \%$ vs. $5.4 \%, \mathrm{P}<0.05$, respectively) and fewer hemodynamic fluctuations after intubation both one and ten minutes after pleural opening.

Conclusions: The use of a BB with the disconnection technique in VATS offers an effective method for improving the quality of lung collapse and reducing postoperative sore throat.

Keywords: One-lung ventilation (OLV); disconnection technique; bronchial blocker (BB); video-assisted thoracoscopic surgery

Submitted Sep 14, 2019. Accepted for publication Dec 05, 2019.

doi: $10.21037 /$ jtd.2019.12.75

View this article at: http://dx.doi.org/10.21037/jtd.2019.12.75 


\section{Introduction}

With the continuous development of thoracic surgery, especially video-assisted thoracoscopic surgery (VATS), onelung ventilation $(\mathrm{OLV})$ is becoming an essential component of thoracic anesthesia (1). The two principal devices used for achieving OLV are a double-lumen tube (DLT) and a bronchial blocker (BB) $(2,3)$.

OLV requires effective nonventilated lung collapse to facilitate surgical exposure. The insertion of a DLT is a well-established technique for achieving OLV; however, since a DLT has a relatively large external diameter and needs to be rotated during the insertion process, the potential risk for traumatic injuries to the airway is high (4). An alternate technique for achieving OLV is the use of a BB (5). The use of a BB causes few hemodynamic fluctuations, and the incidence of postoperative sore throat is low, but some studies have suggested that the use of a $\mathrm{BB}$ takes longer to collapse the operative lung than the use of a DLT, especially in patients with chronic obstructive pulmonary disease (COPD) (5).

Using a two-minute disconnection technique with a DLT has been shown to accelerate the nonventilated lung collapse during OLV in VATS (6). However, the effectiveness of this BB technique in VATS has not been tested systematically, and the proper time for disconnection and the monitoring technique needed have not been provided.

Therefore, the primary aim of this study was to compare the efficacy of a BB with the disconnection technique and a DLT for achieving lung collapse in a randomized, prospective manner. The study further evaluated the safety of and the optimal time needed for the disconnection technique as well as the surgical conditions the technique provides and the postoperative complications.

\section{Methods}

This prospective randomized trial (ChiCTR1900022373) was approved by the Ethics Committee of Fudan University Shanghai Cancer Center (FUSCC), China (Number 1902197-7). From 10 April 2019 to 31 July 2019, patients undergoing scheduled VATS via a left lobectomy or segmentectomy were enrolled in this study. All patients provided written informed consent. These patients were randomized via a computer-generated number sequence into two groups: a group that received a BB with the disconnection technique (Group B) and a group that received a left-sided DLT with spontaneous collapse
(Group D). The inclusion criteria were an age between 20 and 65 years and an American Society of Anesthesiologists (ASA) physical status of I or II. The exclusion criteria were patients with a previously diagnosed or suspected difficult airway, left main bronchus abnormality, history of thoracic surgery or pleural disease, history of COPD or severe asthma, forced expiratory volume in 1 second (FEV1) or forced vital capacity (FVC) less than $50 \%$ of normal values, and communication disorder or mental illness.

The patients received no premedication regimen. After arriving in the operating room, all patients received standard monitoring: pulse oximetry, electrocardiography (ECG), peripheral or central venous access and invasive blood pressure monitoring using the right radial artery. Then, all patients were subjected to the induction of general anesthesia by intravenously administered $0.5 \mu \mathrm{g} / \mathrm{kg}$ sufentanil; the target-controlled infusion (TCI) of $2 \%$ propofol at an effect-site concentration (Ce) of $4 \mu \mathrm{g} / \mathrm{mL}$; and $0.8 \mathrm{mg} / \mathrm{kg}$ rocuronium. After the onset of muscle relaxation and after the propofol Ce reached $2 \mu \mathrm{g} / \mathrm{mL}$, the patients were intubated with a single-lumen endotracheal tube (SLT) or the DLT. In Group D, the patients were intubated using a left-sided DLT (Covidien. Cornamaddy, Co. Westmeath, Athlone, Ireland) of an adequate size (size F35 for women and size F37 for men). The DLT was positioned appropriately using a fiberoptic bronchoscope (FOB). Patients in Group B were intubated using an SLT (male ID $8.0 \mathrm{~mm}$, female $7.5 \mathrm{~mm}$ ) with a depth of 22-24 cm and fixed. Then, the Coopdech BB (Hangzhou Tappa Medical Technology CO., Hangzhou, China) was positioned through the SLT under the guidance of the FOB so that the cuff of the BB was placed in the left main bronchus. An experienced anesthesiologist confirmed the correct placement of either the DLT or the BB. After the patients were turned in order to achieve a right lateral decubitus position, the correct placement of the BB and DLT was reconfirmed using the FOB.

Until the start of surgery, all patients were maintained on two-lung ventilation (TLV), and the cuff of the BB and the bronchial cuff of the DLT were kept deflated. For TLV, mechanical ventilation operated in a volume-controlled mode with a tidal volume of $7-8 \mathrm{~mL} / \mathrm{kg}$ (ideal body weight), a respiratory rate of $10-12$ breaths/min, a positive endexpiratory pressure (PEEP) of $5 \mathrm{cmH}_{2} \mathrm{O}$, and an $\mathrm{FiO}_{2}$ level of 1.0. When the surgeon performed the skin incision, OLV was initiated in both groups. In Group D, the bronchial cuff of the DLT was inflated, and the left channel was opened to the air. In Group B, the lung was deflated via 
the disconnection technique, turning the ventilator off and opening the breathing circuit to the air. No further maneuvers were performed to facilitate lung collapse. Fifteen seconds after opening the pleura and seeing the lung, the cuff of the BB was inflated with 5 to $8 \mathrm{~mL}$ of air to obtain total bronchial, and then ventilation was started again. During OLV, the ventilator settings in both groups were adjusted to keep the peak pressure below $25 \mathrm{cmH}_{2} \mathrm{O}$ by decreasing the tidal volume to $5-7 \mathrm{~mL} / \mathrm{kg}$, and the $\mathrm{FiO}_{2}$ level was adjusted to 0.9 . The ventilation parameters were adjusted to maintain the end-tidal carbon dioxide (ETCO2) level between 35 and $45 \mathrm{mmHg}$. At the end of the surgery, after the lung recruitment maneuver, TLV was maintained with an $\mathrm{FiO}_{2}$ level of 0.5 and a PEEP of $5 \mathrm{cmH}_{2} \mathrm{O}$. When the operation was completed, the patients were transferred to the post-anesthesia care unit (PACU).

The following variables were recorded in this study:

(I) Invasive mean arterial pressure (MAP) and heart rate (HR) before the induction of general anesthesia (T0), immediately after intubation (T1), and $1 \mathrm{~min}$ after intubation (T2); the average of the three values (data in the time point, two seconds before and two seconds after that time point) will be used as the value at that time point.

(II) At 1 and 10 minutes after pleural opening, a surgeon who was blinded to the type of tube being used scored the quality of lung collapse by using a three-point visual and descriptive scale (7) $(1=$ no lung collapse with interference during the surgical procedure; $2=$ partial lung collapse with some residual air in the lung; and $3=$ complete collapse with perfect surgical exposure);

(III) The time required for pleural opening, time required for complete lung collapse, time required for correct placement of the device (measured from the time of intubation to the time the experienced anesthesiologist confirmed the correct placement of either the DLT or the BB), number of patients requiring additional device repositioning during $\mathrm{OLV}$, and the number of patients with hypoxemia $\left(\mathrm{SpO}_{2}<90 \%\right)$ during OLV;

(IV) The number of patients suffering from a sore throat when leaving the PACU and within 24 hours after surgery. The intensity of the sore throat was classified into four levels: no pain, mild (pain with deglutition), moderate (pain present constantly and increasing with deglutition), and severe (pain interfering with eating and requiring analgesic medication).

After randomizing into two groups, the patients, the surgeon scored the quality of lung collapse, the anesthesia nurse in the PACU and for post-operative follow-up were blind to the allocation.

We calculated the sample size based on preliminary results for the first ten patients of each group and estimated that 35 patients in each group would be required to detect a mean difference of $10 \%$ and standard deviation of $10 \%$ with adequate lung collapse and $90 \%$ power at a significance level of $\mathrm{P}<0.05$. We factored in a $10 \%$ dropout rate and enrolled 85 patients in our study. Statistical analyses were performed with SPSS version 17.0 (SPSS Inc., Chicago, IL, USA). The continuous variable is presented as the mean \pm standard deviation (SD). The independent samples $t$-test and the Chi-squared test were used to evaluate the differences between groups in terms of quantitative and qualitative variables, respectively. A $\mathrm{P}$ value $<0.05$ was considered statistically significant (8).

\section{Results}

A total of eighty-five patients were screened for this study. Four patients did not meet the criteria: two patients were younger than 20 years old or older than 65 years, and two patients had FEV $1<65 \%$ of the predicted value. Six patients refused to participate in this study. Therefore, seventy-five patients were subsequently randomly assigned to Group D and Group B (Figure 1). There were no significant differences in the patient characteristics and operative characteristics between the groups $(\mathrm{P}>0.05)$ (Table 1).

\section{Primary outcomes}

Compared with Group D, in Group B, the application of the disconnection technique with the $\mathrm{BB}$ resulted in a similar time for pleural opening and a comparable time required for lung collapse $(\mathrm{P}=0.652,0.226$, respectively, Table 2). Additionally, the quality of lung collapse ten minutes after pleural opening was better than that one minute after pleural opening in all patients; however, there was no significant difference in the quality of lung collapse one and ten minutes after pleural opening between the two groups $(\mathrm{P}=0.621,0.792$, respectively, Figure $2 A, B)$. The intubation time of Group D was significantly longer than that of Group B (2.5 vs. 1.9 min, respectively, $\mathrm{P}=0.007$, Table 2). The number of patients requiring additional device repositioning and the number of patients with hypoxemia 


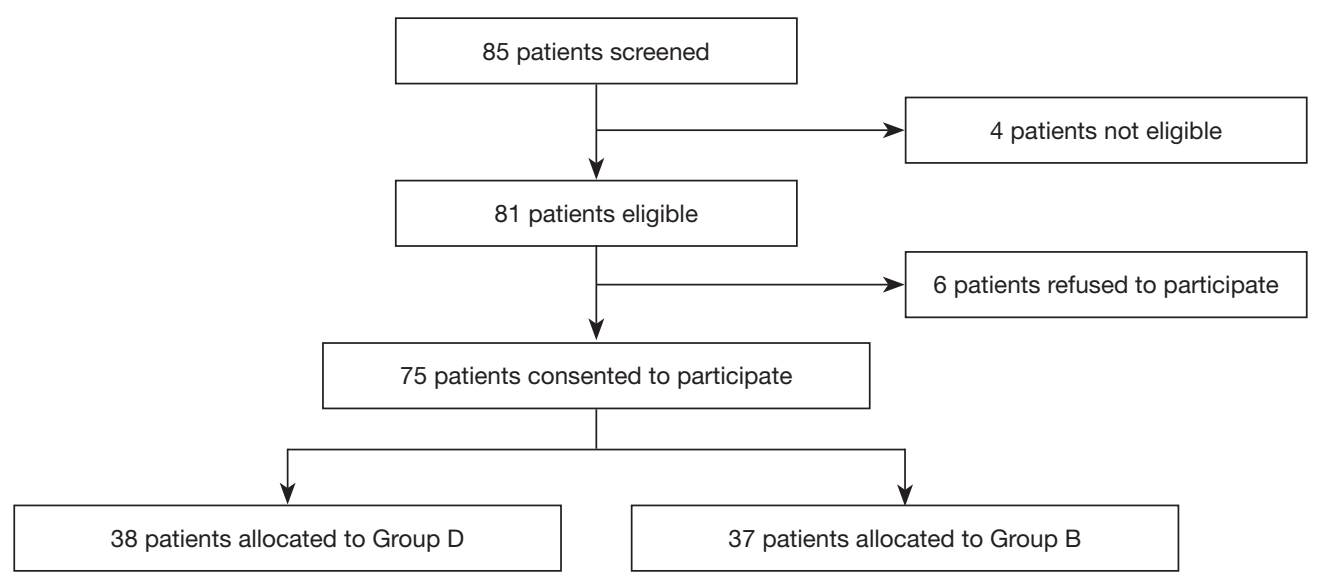

Figure 1 Patient recruitment flow chart.

Table 1 Patient demographic characteristics

\begin{tabular}{lccc}
\hline Variable & $\begin{array}{c}\text { Group D } \\
(\mathrm{n}=38)\end{array}$ & $\begin{array}{c}\text { Group B } \\
(\mathrm{n}=37)\end{array}$ & P value \\
\hline Age (years) & $51.1 \pm 7.3$ & $53.2 \pm 9.1$ & 0.436 \\
Sex (male/female) & $26 / 12$ & $24 / 12$ & 0.872 \\
BMI (kg/m $\left.{ }^{2}\right)$ & $24.2 \pm 3.1$ & $23.4 \pm 4.3$ & 0.360 \\
ASA (I/II) & $13 / 25$ & $13 / 23$ & 0.864 \\
FEV $(\%$ of the predicted value) & $73.2 \pm 5.1$ & $72.5 \pm 6.1$ & 0.593 \\
FVC (\% of the predicted value) & $74.4 \pm 4.3$ & $75.4 \pm 4.5$ & 0.332 \\
Duration of surgery (min) & $105 \pm 25$ & $99 \pm 31$ & 0.361 \\
Duration of anesthesia (min) & $127 \pm 29$ & $124 \pm 27$ & 0.646 \\
Type of surgery ( $\mathrm{n})$ & & & \\
$\quad$ Segmentectomy & 20 & 19 & 0.912 \\
$\quad$ Lobectomy & 18 & 18 & \\
\hline
\end{tabular}

Data are presented as numbers or means \pm standard deviations. ASA, American Society of Anesthesiologists; BMI, body mass index; FEV1, forced expiratory volume in 1 second; FVC, forced vital capacity.

during OLV was similar between the two groups (Table 2).

\section{Secondary outcomes}

The hemodynamic changes that occurred during general anesthesia induction are shown in Figure 3. Compared to T0, at T1, the MAP and HR increased in both groups $(\mathrm{P}>0.05)$. The MAP at T1 in Group D was significantly higher than that at $\mathrm{T} 1$ in Group $\mathrm{B}(\mathrm{P}=0.02)$; furthermore, the HR at T1 in Group D was higher than that at T1 in
Table 2 Clinical outcomes

\begin{tabular}{lccc}
\hline Variable & $\begin{array}{c}\text { Group D } \\
(\mathrm{n}=38)\end{array}$ & $\begin{array}{c}\text { Group B } \\
(\mathrm{n}=37)\end{array}$ & $\mathrm{P}$ value \\
\hline Time required for pleural opening (s) & $87 \pm 15$ & $88 \pm 15$ & 0.652 \\
$\begin{array}{l}\text { Time required for lung collapse }(\min ) \\
\begin{array}{l}\text { Time for placement of the device in } \\
\text { the correct position (min) }\end{array}\end{array}$ & $2.5 \pm 1.3$ & $4.1 \pm 1.6$ & 0.226 \\
$\begin{array}{l}\text { Number of patients requiring } \\
\text { additional device repositioning (n) }\end{array}$ & 3 & 3 & 0.695 \\
$\begin{array}{l}\text { Number of patients with hypoxemia } \\
\text { during OLV }(\mathrm{n})\end{array}$ & 2 & 2 & 0.627 \\
\hline
\end{tabular}

OLV, one-lung ventilation.

Group B, but there was no significant difference between the two groups $(\mathrm{P}=0.18)$.

Compared with patients in Group B, patients in Group $\mathrm{D}$ had a higher incidence of sore throat when leaving the PACU, and most cases were of mild pain $(34.2 \%$ vs. $13.5 \%$, $\mathrm{P}=0.002$, Figure $4 A$ ). In most patients, the sore throat was relieved 24 hours after surgery, but the incidence of mild sore throat was still higher in Group D than in Group B ( $15.8 \%$ vs. $5.4 \%, \mathrm{P}=0.004$, Figure $4 B$ ). No patient suffered from a severe sore throat after surgery.

\section{Discussion}

In this randomized controlled trial, we found that the use of a BB with a disconnection technique could achieve similar lung collapse to the use of a DLT in VATS with fewer slight fluctuations in hemodynamics after intubation, a shorter intubation time and a lower incidence of postoperative sore 

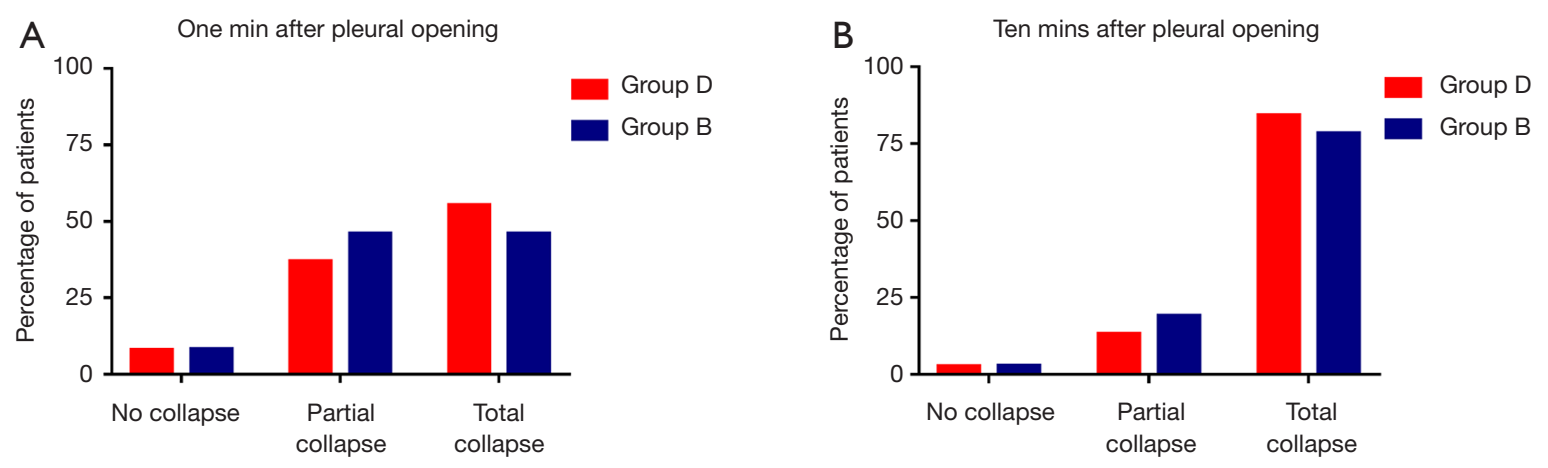

Figure 2 The quality of lung collapse.
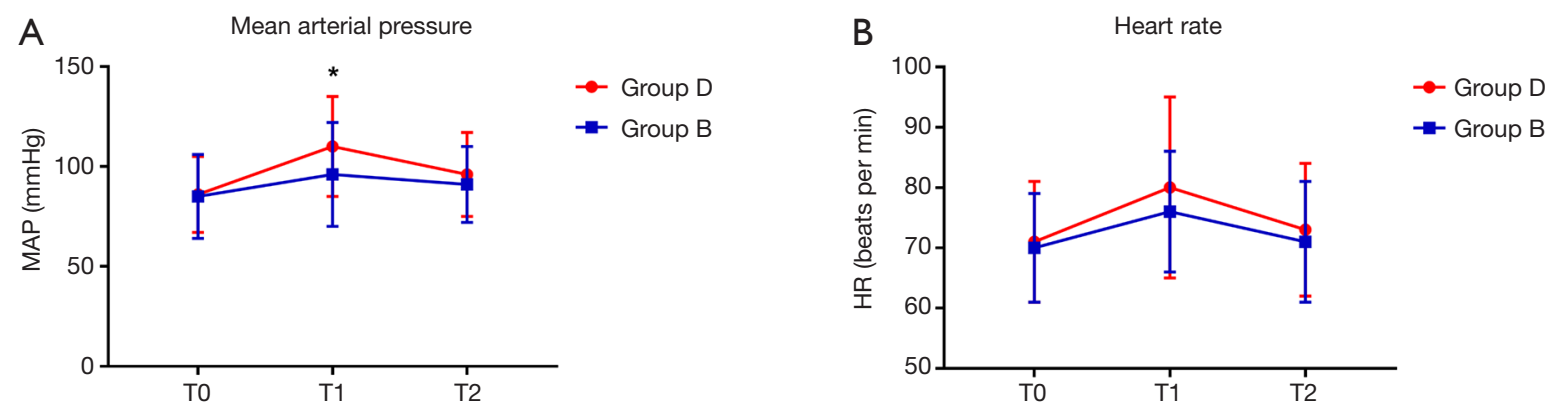

Figure 3 Hemodynamic values during anesthesia induction ( ${ }^{*}, \mathrm{P}<0.05$ compared with Group D).
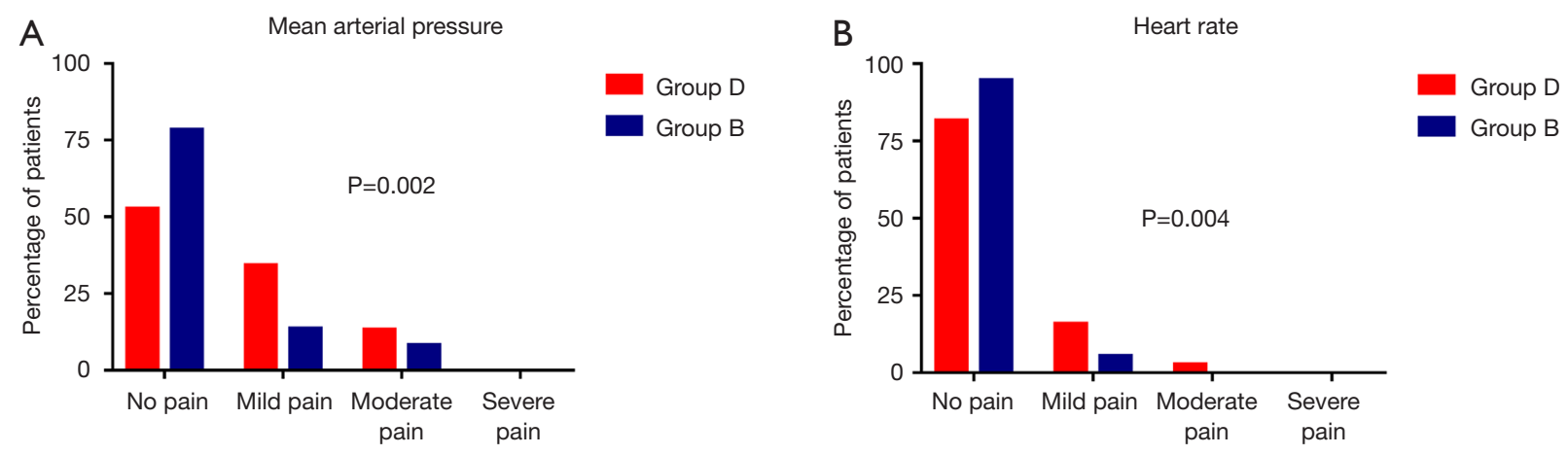

Figure 4 The percentage of patients suffering from sore throat.

throat.

Lung isolation techniques have been extensively used to allow OLV in patients undergoing thoracic surgeries $(9,10)$. OLV requires effective nonventilated lung collapse to facilitate surgical exposure (11). Traditionally, insertion of a DLT has been considered a well-established technique for achieving OLV and has been routine for most anesthesiologists. Some anesthesiologists favor a DLT for its advantages of being quick and easy to place, its convenient CPAP application and its rapid lung collapse $(3,10)$. Our results indicated that for patients undergoing the use of a $\mathrm{BB}$ with the disconnection technique could achieve similar lung collapse to those with the use of a DLT, with a shorter time needed for placement of the device in the correct position. This result may be due to the biphasic nature of lung collapse: a fast collapse phase within the first minute followed by a slow collapse phase during which small airway closure occurs and residual lung gases are 
absorbed $(12,13)$. The disconnection technique with the use of a BB resulted in similar rapid lung collapse in both groups, while the slower collapse phase was not related to the device used. Some methods have been assessed in terms of lung collapse during isolation techniques, such as denitrogenation with an $\mathrm{FiO}_{2}$ of $100 \%$ oxygen using nitrous oxide and suction $(13,14)$. Our study showed that the use of a 15-second disconnection technique with a BB in OLV could accelerate lung collapse and result in excellent lung deflation for satisfactory surgical exposure.

Similarly, a BB has advantages when used for lung isolation, such as that it can be used for patients with a "difficult" airway when a DLT is difficult or impossible to use and that it has limited potential for airway trauma $(15,16)$. Our results indicate that the use of a BB rather than a DLT has slightly smaller hemodynamic interference after intubation, which is beneficial for aged patients or patients with accompanying complicated comorbidities. Additionally, the incidence of postoperative sore throat was significantly lower in Group B than in Group D. In addition, although the disconnection technique with the use of the BB required 15 seconds of ventilation cessation after opening the pleura, there was no significant difference in the incidence of hypoxemia during surgery between the two groups, on account of previous adequate oxygen reserve. This finding indicates that the disconnection technique can be safely used for patients undergoing thoracic surgery.

This study had several limitations. First, the method used to assess lung collapse of a rating scale by a single surgeon was not objective enough. Another limitation was in regards to the patients' characteristics. The recruited patients should have included patients with poor pulmonary function test results. The effect might be more objective when all pulmonary function patients are included.

\section{Conclusions}

The disconnection technique with the use of a BB is an effective method for improving the time and quality of nonventilated lung collapse in VATS and results in overall lung collapse comparable with the use of a DLT. This method offers an effective technique for accelerating lung collapse.

\section{Acknowledgments}

Funding: This research was supported by the National Natural Science Foundation of China (No. 81873948,
81871590, 81871591), the National Key Research and Development Program of China (No. 2018YFC2001904), Shanghai Shenkang Hospital Development Center Clinical Science and Technology Innovation Project (No. SHDC12018105), the Key Technology and Development Program of Shanghai (No. 17411963400).

\section{Footnote}

Conflicts of Interest: WC serves as the unpaid section editor of Fournal of Thoracic Disease. The other authors have no conflicts of interest to declare.

Ethical Statement: The authors are accountable for all aspects of the work in ensuring that questions related to the accuracy or integrity of any part of the work are appropriately investigated and resolved. This prospective randomized trial (ChiCTR1900022373) was approved by the Ethics Committee of Fudan University Shanghai Cancer Center (FUSCC), China (Number 1902197-7). All patients provided written informed consent.

Open Access Statement: This is an Open Access article distributed in accordance with the Creative Commons Attribution-NonCommercial-NoDerivs 4.0 International License (CC BY-NC-ND 4.0), which permits the noncommercial replication and distribution of the article with the strict proviso that no changes or edits are made and the original work is properly cited (including links to both the formal publication through the relevant DOI and the license). See: https://creativecommons.org/licenses/by-nc-nd/4.0/.

\section{References}

1. Brodsky JB, Lemmens HJ. The history of anesthesia for thoracic surgery. Minerva Anestesiol 2007;73:513-24.

2. Lu Y, Dai W, Zong Z, et al. Bronchial Blocker Versus Left Double-Lumen Endotracheal Tube for One-Lung Ventilation in Right Video-Assisted Thoracoscopic Surgery. J Cardiothorac Vasc Anesth 2018;32:297-301.

3. Falzon D, Alston RP, Coley E, et al. Lung Isolation for Thoracic Surgery: From Inception to Evidence-Based. J Cardiothorac Vasc Anesth 2017;31:678-93.

4. Knoll H, Ziegeler S, Schreiber JU, et al. Airway injuries after one-lung ventilation: a comparison between double-lumen tube and endobronchial blocker: a randomized, prospective, controlled trial. Anesthesiology 2006; $105: 471-7$. 
5. Clayton-Smith A, Bennett K, Alston RP, et al. A Comparison of the Efficacy and Adverse Effects of Double-Lumen Endobronchial Tubes and Bronchial Blockers in Thoracic Surgery: A Systematic Review and Meta-analysis of Randomized Controlled Trials. J Cardiothorac Vasc Anesth 2015;29:955-66.

6. Li Q, Zhang X, Wu J, et al. Two-minute disconnection technique with a double-lumen tube to speed the collapse of the non-ventilated lung for one-lung ventilation in thoracoscopic surgery. BMC Anesthesiol 2017;17:80.

7. Ruetzler K, Grubhofer G, Schmid W, et al. Randomized clinical trial comparing double-lumen tube and EZBlocker for single-lung ventilation. Br J Anaesth 2011;106:896-902.

8. Zhang Z. Univariate description and bivariate statistical inference: the first step delving into data. Ann Transl Med 2016;4:91.

9. Campos JH. Progress in lung separation. Thorac Surg Clin 2005; 15:71-83.

10. Bernasconi F, Piccioni F. One-lung ventilation for thoracic surgery: current perspectives. Tumori 2017;103:495-503.
11. Campos JH. Current techniques for perioperative lung isolation in adults. Anesthesiology 2002;97:1295-301.

12. Pfitzner J, Peacock MJ, Harris RJ. Speed of collapse of the non-ventilated lung during single-lung ventilation for thoracoscopic surgery: the effect of transient increases in pleural pressure on the venting of gas from the nonventilated lung. Anaesthesia 2001;56:940-6.

13. Quan X, Yi J, Huang Y, et al. Bronchial suction does not facilitate lung collapse when using a double-lumen tube during video-assisted thoracoscopic surgery: a randomized controlled trial. J Thorac Dis 2017;9:5244-8.

14. Yoshimura T, Ueda K, Kakinuma A, et al. Bronchial blocker lung collapse technique: nitrous oxide for facilitating lung collapse during one-lung ventilation with a bronchial blocker. Anesth Analg 2014;118:666-70.

15. Neustein SM. Pro: bronchial blockers should be used routinely for providing one-lung ventilation. J Cardiothorac Vasc Anesth 2015;29:234-6.

16. Collins SR, Titus BJ, Campos JH, et al. Lung Isolation in the Patient With a Difficult Airway. Anesth Analg 2018;126:1968-78.
Cite this article as: Cheng Q, He Z, Xue P, Xu Q, Zhu M, Chen W, Miao C. The disconnection technique with the use of a bronchial blocker for improving nonventilated lung collapse in video-assisted thoracoscopic surgery. J Thorac Dis 2020;12(3):876-882. doi: 10.21037/jtd.2019.12.75 